\title{
Ferramenta para Mediação do Processo de Desenvolvimento do Pensamento Algorítmico contemplando Preceitos de Acessibilidade
}

\author{
Cristina Paludo Santos, Vinicius Ajala, Denilson Rodrigues da Silva \\ Universidade Regional Integrada do Alto Uruguai e das Missões (URI) \\ 98.802-470 - Santo Ângelo - RS - Brasil \\ paludo@san.uri.br, viniciusajala@outlook.com, deniro@san.uri.br
}

\begin{abstract}
This paper presents CCEduc - a computational tool that aims to support the process of algorithmic thinking development and to concretize principles of equity promoting spaces of sociodigital mediation for blind individuals. Designed in accordance with the principles of accessibility, it is intended, with this article, to present the strategies implemented in the modeling of the tool to enhance its use in the construction of knowledge and to promote practices of technological mediation for inclusion.
\end{abstract}

Resumo. Este artigo apresenta a ferramenta CCEduc, que visa apoiar o processo de desenvolvimento do pensamento algorítmico e concretizar princípios de equidade promovendo espaços de mediação para interagentes cegos. Projetada em sintonia com os princípios de acessibilidade pretende-se, com este artigo, apresentar as estratégias implementadas na modelagem da ferramenta para potencializar o seu uso na construção do conhecimento e promover práticas de mediação tecnológica para a inclusão.

\section{Introdução}

O estabelecimento de uma base metodológica para a educabilidade da diversidade humana incitou a necessidade de reavaliar planos e métodos para a construção de sistemas computacionais com vistas a imprimir o conceito da diferença [Sonza, 2008; Baranauskas, 2012; Lima, 2017].

Nesta perspectiva em que busca-se concretizar princípios de equidade na configuração dos recursos computacionais se insere este trabalho. A proposta contempla a concepção e garantia de acessibilidade na ferramenta computacional CCEduc com ênfase na aprendizagem de programação que auxilia no processo de desenvolvimento do pensamento algorítmico [Ajala, 2016]. A ferramenta visa fortalecer os métodos de ensino para formação de competências de acadêmicos de computação, também busca imprimir a força da diferença em sua interface para potencializar a interação, o desenvolvimento, a sedimentação da teoria e obtenção de melhores resultados no aprendizado de interagentes cegos.

A ferramenta estabelece um elo entre a aprendizagem de algoritmos, qualidade de software e recursos de acessibilidade e provê funcionalidades para comparar uma solução algorítmica desenvolvida por um aluno a uma solução referencial na literatura, usando como critério principal nos resultados a análise da complexidade ciclomática. Considerando a dimensão pedagógica, sua estratégia de concepção busca superar o 
VIII Congresso Brasileiro de Informática na Educação (CBIE 2019)

Anais do XXV Workshop de Informática na Escola (WIE 2019)

instrucionismo e ratificar projetos de aprendizagem como fio condutor do processo de desenvolvimento tornando o ensino do conteúdo abordado mais prático e abrangente, de forma a despertar o interesse do aluno, o seu espírito de pesquisa e a busca de informações que possam torná-lo um profissional crítico e de opinião sólida.

Uma descrição das dimensões tecnopedagógicas da ferramenta é apresentada nas próximas seções. A seção 2 apresenta os principais conceitos que nortearam o desenvolvimento da ferramenta, bem como alguns trabalhos relacionados. A seção 3 apresenta as principais características da ferramenta CCEduc. A seção 4 apresenta os recursos de acessibilidade contemplados. A seção 5 descreve o processo de validação da ferramenta quanto à funcionalidade e ao conjunto de recursos que compõe a interface. Por fim, a seção 6 apresenta as considerações finais e perspectivas de trabalhos futuros.

\section{O Pensamento Algorítmico e a Complexidade Ciclomática}

Um dos maiores desafios que se impõe ao aluno durante o processo de formação acadêmico profissional é o de deixar de ser um mero utilizador ou executor de programas. Antes de ver o mundo como um conglomerado de desafios computacionais é necessário visualizar e assimilar os problemas como uma estrutura que pode ser solucionada, isto é, o aluno precisa desenvolver faculdades mentais de modo a tornar-se um indivíduo crítico, para depois então dirimir os problemas da sociedade com sua especialidade [Wing, 2014]. É neste contexto que surge a aptidão que é vista como imprescindível - o pensamento algorítmico [Souza, 2016; Rodriguez, 2017].

Uma das premissas para se estabelecer o pensamento computacional é a preocupação pela melhor solução possível. Esta é a base do pensamento algorítmico que expressa soluções em diferentes passos de forma a encontrar uma saída mais eficiente e eficaz de resolver um problema [Resnick 2012]. O pensamento algorítmico é um ramo do pensamento computacional, sendo uma representação mais abstrata da forma de se pensar. Não é ensinado diretamente na graduação como uma área de estudo, mas sim de forma indireta quando se coloca o aluno em situações de aprendizagem de lógica de programação por meio de fatos do cotidiano, para depois formular soluções através de procedimentos computacionais.

A complexidade ciclomática é uma métrica cujo objetivo é medir a complexidade de determinado código fonte. Quanto maior o seu valor, maior a dificuldade de se entender, modificar e, consequentemente, testar o código fonte [McCabe 1976]. De forma a facilitar a visualização do fluxo de um código fonte, McCabe utiliza o conceito de Grafo de Fluxo de Controle como forma de representação, composto por vértices que fazem referência a blocos ou linhas de código fonte, e arestas que conectam os vértices formando caminhos do fluxo de execução [Allen 1970], identificando quais são os possíveis caminhos de execução de um algoritmo.

Neste trabalho, a complexidade ciclomática é empregada de forma inovadora no contexto de aprendizagem algorítmica, servindo de apoio no processo inicial de constituição do pensamento algorítmico, possibilitando o seu acesso para alunos cegos.

\section{A Ferramenta CCEduc}

A modelagem da ferramenta CCEduc respalda-se nos princípios de praticidade e apoio à etapa de aprendizagem de programação básica, buscando refletir melhores soluções 
VIII Congresso Brasileiro de Informática na Educação (CBIE 2019)

Anais do XXV Workshop de Informática na Escola (WIE 2019)

perante o aluno. De forma geral, a concepção da ferramenta considera a ideia de que o aluno forneça a solução de um desafio a ele dado (algoritmo). Após sua análise é gerado o grafo ciclomático da solução do aluno e da solução armazenada na base de conhecimento, elucidando assim uma comparação gráfica que permite abstrair os conceitos de complexidade algorítmica.

O grafo facilita a visualização do fluxo de um código, sendo composto por vértices que fazem referência a blocos ou linhas de código e arestas que conectam os vértices formando caminhos do fluxo de execução, identificando quais são os possíveis caminhos de execução de um algoritmo. A proposta atenta para o uso de indicadores quantitativos abordados para o aluno de forma qualitativa como, por exemplo, arestas e vértices representados por um grafo que visa refletir conceitos de qualidade de software.

O processo de concepção da ferramenta envolveu as seguintes etapas: (a) a definição de uma base de conhecimento que armazena uma coleção de códigos que se constitui em soluções apontadas como referências para determinados algoritmos; (b) a extração de estruturas de desvio de fluxo do código-fonte para construção do grafo da complexidade ciclomática de ambos códigos-fonte (do aluno e do referencial); (c) a definição dos componentes do grafo (vértices, arestas e coordenadas dos vértices); (d) implementação do cálculo do número ciclomático em que utilizou-se a fórmula: $\mathrm{CC}=\mathrm{A}$ - V +2 [McCabe 1976] para o cálculo da complexidade, onde CC é o número ciclomático, $\mathrm{A}$ é o número de arestas e $\mathrm{V}$ é o número de vértices e, (e) comparação das soluções com retorno de indicadores ao aluno.

A ferramenta foi codificada na linguagem Java e desenvolvida conforme o padrão MVC. Uma descrição mais detalhada de cada um dos componentes que formam o modelo da ferramenta pode ser obtida em Ajala [Ajala, 2016].

\section{Recursos de Acessibilidade Contemplados na Ferramenta CCEduc}

Sedimentado em uma abordagem pragmática, a pesquisa acerca-se do efeito prático e benéfico que a concepção de um artefato tecnológico possa ser capaz de gerar para a solução de problemas. Essa abordagem possibilita dimensionar o uso de tecnologias emergentes no processo de desenvolvimento de artefatos de cunho assistivo. Metodologicamente, o desenvolvimento da interface $\mathrm{e}$ das funcionalidades $\mathrm{da}$ ferramenta CCEduc envolveu a incorporação de recursos de acessibilidade baseados no sistema TTS ("Text To Speech") que transforma um texto simples em voz. Tal sistema de interação mostra-se útil visto que pode ser acoplado como um módulo em sistemas de diálogo e, também, ser utilizado como leitor de tela para interagentes cegos.

Utilizou-se a biblioteca FurbSpeech que gera arquivos de texto contendo uma série de símbolos fonéticos, juntamente com sua frequência, duração e amplitude. Para transformar os arquivos de fonemas em som propriamente dito, foi utilizado um sintetizador chamado MBROLA. Ele recebe como entrada um arquivo de fonemas e gera um arquivo de áudio no formato WAV. Para que houvesse a integração entre a biblioteca do Furbspeech, foi criado um pacote chamado Synthetizer.mbrola dentro do projeto. Este pacote é responsável por armazenar os arquivos que serão sintetizados. Na classe principal do projeto onde é executado o método main, foi criada uma classe chamada FurbSpeechText, com um método toText( ). Este método é o responsável por receber uma String, e fazer a sintetização para a fala. Logo, em praticamente todo o 
VIII Congresso Brasileiro de Informática na Educação (CBIE 2019)

Anais do XXV Workshop de Informática na Escola (WIE 2019)

projeto, existem objetos da classe FurbSpeechText sendo instanciados, e repassando uma string para o método toText.

A interface acessível da ferramenta proporciona a apresentação em áudio da descrição dos elementos que compõem as soluções algorítmicas, como número ciclomático, número de arestas, vértices, regiões e estruturas de desvio, tanto da solução referencial como do usuário. Essa descrição é a responsável pelas informações capazes de provocar a construção de uma imagem mental dos conceitos do diagrama representado visualmente de maneira semelhante ao que ocorre durante a leitura tátil. A Tabela 1 apresenta as combinações de teclas definidas como atalhos para contemplar a interação no modo assistivo.

Tabela 1. Teclas de atalho na ferramenta CCEduc

\begin{tabular}{|c|l|}
\hline Tecla de Atalho & \multicolumn{1}{|c|}{ Descrição } \\
\hline F1 & $\begin{array}{l}\text { Ajuda, explica em áudio como utilizar a } \\
\text { ferramenta no modo assistivo }\end{array}$ \\
\hline F4 & $\begin{array}{l}\text { Fechar janela de resultados e voltar a tela } \\
\text { principal }\end{array}$ \\
\hline F9 & Ouvir os algoritmos antes de soluciona-los \\
\hline F10 & Analisar o código \\
\hline F11 & Dar ok após inserir o código \\
\hline F12 & $\begin{array}{l}\text { Entrar no modo assistivo ao iniciar a } \\
\text { ferramenta }\end{array}$ \\
\hline Ctr1 + I & Inserir código (solução) \\
\hline Ctr1 + X & Sair da ferramenta \\
\hline
\end{tabular}

A ferramenta foi concebida de modo que tanto interagentes cegos quanto videntes possam utilizar os mesmos recursos. Para a avaliação dos recursos de acessibilidade contemplados na ferramenta, contou-se com a participação de 5 avaliadores sendo 2 deles docentes atuantes na disciplina de Interação HumanoComputador, 1 pesquisador na área de Tecnologias Assistivas e 2 professores da Educação Especial. Foram propostos cenários de uso a fim de analisar o atendimento das seguintes premissas: (a) é possível o acesso a todas as funcionalidades via teclado comum; (b) a interação com a ferramenta se dá mediante áudio compreensível; (c) é possível navegar sem a presença de um instrutor.

Todos os participantes concordaram que não há ruídos ou atrasos no áudio e que é possível navegar sem a presença de um instrutor uma vez que as instruções fornecidas são claras e objetivas. No entanto, embora a maioria das funcionalidades da ferramenta possam ser acessadas via teclas de atalho, 3 dos avaliadores detectaram limitações de acesso a determinadas funções que consideram importantes, tais como: informar ao usuário sobre a sua posição atual; possibilitar ouvir novamente o resultado das análises, tanto no que se refere aos grafos quanto aos indicadores numéricos. Tais apontamentos estabelecem a necessidade de aprimoramentos na interface.

\section{Considerações Finais}

Este artigo apresentou uma ferramenta tecnológica acessível que provê recursos para auxiliar o desenvolvimento do pensamento computacional por meio da otimização de soluções algorítmicas. Devido à amplitude que a tríade educação-tecnologiaacessibilidade alcança, este trabalho está restrito às questões de acessibilidade para interagentes cegos, provendo autonomia a um estudante cego no uso da ferramenta e 
VIII Congresso Brasileiro de Informática na Educação (CBIE 2019)

Anais do XXV Workshop de Informática na Escola (WIE 2019)

possibilitando que estes possam interagir com seus colegas videntes em um trabalho em grupo a partir do acesso aos mesmos recursos.

Cabe destacar que a evolução gradativa da ferramenta estabelece a necessidade do estudo e incorporação de novos recursos de acessibilidade, tanto para promover o acesso às suas novas funcionalidades, quanto para ampliar as possibilidades do uso da ferramenta para interagentes que possuam outras limitações visuais como, por exemplo, baixa visão e daltonismo. Estudos neste sentido estão sendo desenvolvidos visando contemplar recursos como lupa, alto contraste, resolução de cores e edição de fontes, ampliando assim as potencialidades de interação na construção do conhecimento. Além disso, uma avaliação da ferramenta com alunos cegos também deverá ser realizada.

\section{Referências Bibliográficas}

Ajala, Vinicius; Silva, Denilson Rodrigues; Santos, Cristina Paludo; Rolim, Carlos Oberdan. Análise da Complexidade Ciclomática como Apoio ao Processo de Desenvolvimento do Pensamento Algorítmico. In: Anais do XXXVI Congresso da Sociedade Brasileira de Computação - $5^{\circ}$ Workshop de Desafios da Computação Aplicada à Educação (DesafiE). SBC: Porto Alegre, 2016, p. 587-596.

Baranaukas, Maria Cecília C.; Mantoan, Maria Teresa Eglér. Acessibilidade em ambientes educacionais: para além das guidelines. Educação Temática Digital, v. 2, n. 2, p. 13, 2012.

Costa, António Pedro; Da Costa, Estela Barreto. Contributos para o Desenvolvimento de Software Educativo tendo por base Processos Centrados no Utilizador. Em Teia| Revista de Educação Matemática e Tecnológica Iberoamericana-ISSN: 2177-9309, v. 4, n. 2, 2013.

Lima, Igor et al. SpaceEduc: Uma Proposta para Estimular a Aprendizagem de Alunos Portadores de Necessidades Educacionais Especiais. In: Brazilian Symposium on Computers in Education (Simpósio Brasileiro de Informática na Educação-SBIE). 2017. p. 1751.

McCabe, Thomas J. (1976). A Complexity Measure. Department of Defense, National Security Agency - EUA.

Resnick, M. Brennan (2012). New frameworks for studying and assessing the development of computational thinking. American Educational Research Association meeting. Vancouver $\mathrm{BC}$ - Canadá.

Rodriguez, Carla et al. Met@ Aprendiz@ gem: estratégias metacognitivas e recursos pedagógicos para apoiar a construção do pensamento lógico. In: Brazilian Symposium on Computers in Education (Simpósio Brasileiro de Informática na Educação-SBIE). 2017. p. 1775 .

Savi, Rafael; De Souza, Caroline Battistello Cavalheiro. Design centrado no usuário e o projeto de soluções educacionais. Revista E-Tech: Tecnologias para Competitividade Industrial-ISSN-1983-1838, p. 33-52, 2015.

Sonza, Andréa Poletto; Santarosa, Lucila; Conforto, Débora. Ambientes Virtuais Acessíveis sob a perspectiva de usuários deficientes visuais. In: Brazilian Symposium on Computers in Education (Simpósio Brasileiro de Informática na Educação-SBIE). 2008. p. 74-83.

Souza, Draylson Micael; Da Silva Batista, Marisa Helena; Barbosa, Ellen Francine. Problemas e Dificuldades no Ensino e na Aprendizagem de Programação: Um Mapeamento Sistemático. Revista Brasileira de Informática na Educação, v. 24, n. 1, 2016.

Wing, J. Computational thinking benefits society. 40th Anniversary Blog of Social Issues in Computing, v. 2014, 2014. 